\title{
Colistin as a first choice antibiotic for the initial empiric antimicrobial therapy of ventilator-associated pneumonia
}

\section{To the Editors:}

We read with great interest the article recently published in the European Respiratory Journal (ERJ) by RIOs et al. [1] regarding ventilator-associated pneumonia (VAP) due to colistin susceptible-only microorganisms. The authors concluded that colistin could be a suitable antibiotic for the initial empiric antimicrobial therapy in their intensive care unit (ICU) since they identified 30 carbapenem-sensitive (Carb-S) and 31 colistin-sensitive (Col-S) isolates.

In our seven-bed general ICU 184 patients were admitted during a period of 18 months with the following characteristics: $125(67.9 \%)$ males; age (mean $\pm \mathrm{SD}) 57.8 \pm 22.7 \mathrm{yrs}$; Simplified Acute Physiology Score II 48.2 \pm 11.8 ; Acute Physiology and Chronic Health Evaluation II 19.1 \pm 5.7 ; mean ICU stay $36.7 \pm 36.9$ days; mean mechanical ventilation duration 29.2 \pm 15.9 days; and a total of 3,262 ventilator days.

A total of 52 (28.3\%) VAP episodes (15.9 episodes per 1,000 ventilator days, with a mean clinical pulmonary infection score at VAP onset of $7.4 \pm 1.6)$ were identified, of which 13 $(25 \%)$ were early- and 39 (75\%) late-onset VAP. The mortality rate was $19 \%$, i.e. 10 out of 52 patients, of which: four were attributed to inappropriate treatment; five to delayed antibiotic treatment; and one to inappropriate and delayed treatment.

We had 56 (four patients with two pathogens) isolates from respiratory specimen cultures, all of them Gram-negative microorganisms: 45 Acinetobacter baumannii, nine Pseudomonas aeruginosa and two Klebsiella pneumoniae. Susceptibility results are shown in table 1.

According to our results, we had 50 Carb-S and only six Col-S isolates, which is a significant difference compared with the study by Rios et al. [1]. Also, only eight of the 50 Carb-S isolates were sensitive to imipenem but all 50 were meropenem sensitive (Mer-S). Another difference with that study [1] is that 41 gentamicin-sensitive (Gent-S) isolates were identified.

We also found that late-onset VAP due to P. aeruginosa may be Col-S and, in part, sensitive to piperacilline-tazobactam, while early-onset VAP due to $P$. aeruginosa is multisensitive. Conversely, pneumonia due to $A$. baumannii, even in early VAP, may be mainly due to multidrug-resistant species (41 out of 45) and specifically Gent-S, Mer-S and Col-S (35, 43 and 45 out of 45 patients, respectively).

Summarising our results, out of 56 isolates, 10 were multisensitive isolates, 41 were Gent-S, 50 Mer-S and 56 Col-S, with only six of the 56 isolates identified as colistin-susceptible only.

According to our ecology and sensitivities and despite several differences regarding isolated pathogens and sensitivities compared with that study [1], colistin should be considered as an antibiotic for the initial empiric management of VAP. Gentamicin could not be used as an alternative choice in empiric treatment as it covers only $73.2 \%$ of the isolates. Using

TABLE 1 Susceptibility results for microorganisms in early and late ventilator-associated pneumonia

Total $n$

Susceptibilities

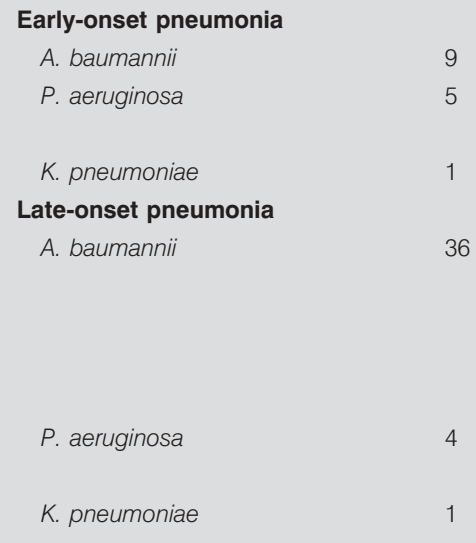


colistin with or without meropenem, all possible pathogens can be covered and then treatment de-escalated according to susceptibility results.

This practice is in accordance with the American Thoracic Society (ATS)/Infectious Diseases Society and America (IDSA) guidelines [2], which recommend consideration of local microbiological data when selecting empiric treatment for VAP and that each hospital and ICU should ideally have their own antibiograms, which should be updated as often as possible. The ATS/IDSA guidelines [2] suggest also considering colistin for the management of VAP attributed to carbapenem-resistant Acinetobacter spp. Also, it has been suggested that each ICU should design specific empiric treatment protocols that are likely to be effective according to local microbiology and resistance patterns [3]. Finally, and according to this concept, BEARDSLEY et al. [4] recently published a significant article introducing the first institutionspecific developed guidelines for the management of hospitalacquired pneumonia.

It is important to note the daily costs of meropenem ( 2 gr $\times 3=6$ gr i.v. daily; AstraZeneca, Macclesfield, UK) and colistin therapy ( 3 million IU $\times 3=9$ million i.v. daily; Norma, Athens, Greece), which are $€ 241,032$ and $€ 13,68$, respectively! Also we would like to inform the readers of the ERJ that we did not experience significant adverse effects with colistin use, i.e. nephrotoxicity requiring discontinuation of the drug or dialysis, except a transient elevation of creatinine $\leqslant 1.6 \mathrm{mg} \cdot \mathrm{dL}^{-1}$ in three patients, which was normalised after adjustment of colistin dosing to creatinine clearance.
In conclusion, we totally agree with Rios et al. [1] suggesting colistin as a suitable antibiotic for the initial empiric antimicrobial therapy in intensive care units with carbapenem resistant pathogens.

\section{K. Ioannides, P. Myrianthefs and G. Baltopoulos}

Athens University School of Nursing, KAT General Hospital, Athens, Greece.

\section{STATEMENT OF INTEREST}

None declared.

\section{REFERENCES}

1 Rios FG, Luna CM, Maskin B, et al. Ventilator-associated pneumonia due to colistin susceptible-only microorganisms. Eur Respir J 2007; 30: 307-313.

2 American Thoracic Society, Infectious Diseases Society of America. Guidelines for the management of adults with hospital-acquired, ventilator-associated, and healthcareassociated pneumonia. Am J Respir Crit Care Med 2005; 171: 388-416.

3 Torres A, Ewig S. Diagnosing ventilator-associated pneumonia. N Engl J Med 2004; 350: 433-435.

4 Beardsley JR, Williamson JC, Johnson JW, Ohl CA, Karchmer TB, Bowton DL. Using local microbiologic data to develop institution-specific guidelines for the treatment of hospital-acquired pneumonia. Chest 2006; 130: 787-793.

DOI: $10.1183 / 09031936.00102207$ 